\title{
Virial relations for the Dirac equation and their applications to calculations of $\mathrm{H}$-like atoms
}

\author{
V. M. Shabaev \\ Department of Physics, St. Petersburg State University, \\ Oulianovskaya Street 1, Petrodvorets, St. Petersburg 198504, Russia
}

\begin{abstract}
Virial relations for the Dirac equation in a central field and their applications to calculations of H-like atoms are considered. It is demonstrated that using these relations allows one to evaluate various average values for a hydrogenlike atom. The corresponding relations for non-diagonal matrix elements provide an effective method for analytical evaluations of infinite sums that occur in calculations based on using the reduced Coulomb-Green function. In particular, this method can be used for calculations of higher-order corrections to the hyperfine splitting and to the $g$ factor in hydrogenlike atoms.
\end{abstract}

\section{Introduction}

In non-relativistic quantum mechanics, the virial theorem for a particle moving in a central field $V(r)$ is given by the well known equation:

$$
\langle T\rangle=\frac{1}{2}\left\langle r \frac{d V}{d r}\right\rangle,
$$

where $\langle T\rangle$ denotes the average value of the kinetic energy in a stationary state. This theorem can easily be derived from the equation $[1]$

$$
\frac{d}{d t}\langle A\rangle=\frac{i}{\hbar}\langle[H, A]\rangle=0,
$$

if we take $A=(\mathbf{r} \cdot \mathbf{p})$. Equation (2), which is generally called as the hypervirial theorem, can also be employed to derive virial relations for diagonal matrix elements of other operators [2]. An extention of these relations to the case of non-diagonal matrix elements was considered in $[3,4]$.

For the Dirac equation in a central field, the virial theorem was first derived by Fock [5]. If we denote the bound-state energy by $E$, it gives

$$
E=\left\langle m c^{2} \beta\right\rangle+\left\langle r \frac{d V}{d r}\right\rangle+\langle V\rangle,
$$

where $\beta$ is the Dirac matrix. For the Coulomb field, one easily finds

$$
E=\left\langle m c^{2} \beta\right\rangle .
$$


Some additional virial relations for the Dirac equation were obtained by a number of authors (see, e.g., $[2,6]$ and references therein). Virial relations, which yield recurrence formulas for various average values, were obtained in $[2,7,8,9]$. In the case of the Coulomb field, these relations can be employed to derive explicit formulas for the average values $\left\langle r^{s}\right\rangle,\left\langle r^{s} \beta\right\rangle$, and $\left\langle i^{s}(\boldsymbol{\alpha} \cdot \mathbf{n}) \beta\right\rangle$, where $\boldsymbol{\alpha}$ is a vector incorporating the Dirac matrices, $\mathbf{n}=\mathbf{r} / r$, and $s$ is integer. The corresponding recurrence relations for non-diagonal matrix elements were derived in [9]. In the case of the Coulomb field, it was found that these relations can be employed to derive explicit formulas for the first-order corrections to the Dirac wave function due to interaction with perturbative potentials of the form $\sim r^{s}, r^{s} \beta, r^{s}(\boldsymbol{\alpha} \cdot \mathbf{n})$, and $i r^{s}(\boldsymbol{\alpha} \cdot \mathbf{n}) \beta$. Later on (see references below), this method was used for calculations of various corrections to the energy levels, to the hyperfine structure splitting, and to the bound-electron $g$ factor. In constract to direct analytical and numerical calculations, the virial relation method allows one to derive formulas for various physical quantities by simple algebraic transformations.

In the present paper, following mainly to [9], we derive the virial relations for the Dirac equation and examine their applications to calculations of H-like atoms. Relativistic units $(\hbar=c=1)$ are used in the paper.

\section{Derivation of the virial relations for the Dirac equa- tion}

For the case of a central field $V(r)$, the Dirac equation has the form

$$
(-i \boldsymbol{\alpha} \cdot \boldsymbol{\nabla}+\beta m+V(r)) \psi(\mathbf{r})=E \psi(\mathbf{r}) .
$$

The wave function is conveniently represented by

$$
\psi(\mathbf{r})=\left(\begin{array}{c}
g(r) \Omega_{\kappa m}(\mathbf{n}) \\
i f(r) \Omega_{-\kappa m}(\mathbf{n})
\end{array}\right)
$$

where $\kappa=(-1)^{j+l+1 / 2}(j+1 / 2)$ is the quantum number determined by the angular momentum and the parity of the state. Substituting this expression into (5), we obtain the radial Dirac equations

$$
\begin{aligned}
& \frac{d G}{d r}+\frac{\kappa}{r} G-(E+m-V) F=0, \\
& \frac{d F}{d r}-\frac{\kappa}{r} F+(E-m-V) G=0,
\end{aligned}
$$

where $G(r)=r g(r)$ and $F(r)=r f(r)$. Introducing the operator [10]

$$
H_{\kappa}=-i \sigma_{y} \frac{d}{d r}+\sigma_{x} \frac{\kappa}{r}+\sigma_{z} m+V,
$$

where $\sigma_{x}, \sigma_{y}$, and $\sigma_{z}$ are the Pauli matrices, and denoting

$$
\phi(r)=\left(\begin{array}{c}
G(r) \\
F(r)
\end{array}\right)
$$


we obtain

$$
H_{\kappa} \phi=E \phi .
$$

The operator $H_{\kappa}$ is self-adjoint in the space of two-component functions satisfying the boundary conditions

$$
\phi(0)=\phi(\infty)=0 .
$$

The scalar product in this space is defined by

$$
\langle a \mid b\rangle=\int_{0}^{\infty} d r\left(G_{a} G_{b}+F_{a} F_{b}\right) .
$$

Let us denote the eigenvalues and the eigenvectors of the operator $H_{\kappa}$ by $E_{n \kappa}$ and $\phi_{n \kappa}$, respectively, where $n$ is the principal quantum number. Taking into account the selfadjointness of $H_{\kappa}$, we can write down the following equations

$$
\begin{aligned}
& \left\langle n^{\prime} \kappa^{\prime}\left|\left(H_{\kappa^{\prime}} Q-Q H_{\kappa}\right)\right| n \kappa\right\rangle=\left(E_{n^{\prime} \kappa^{\prime}}-E_{n \kappa}\right)\left\langle n^{\prime} \kappa^{\prime}|Q| n \kappa\right\rangle, \\
& \left\langle n^{\prime} \kappa^{\prime}\left|\left(H_{\kappa^{\prime}} Q+Q H_{\kappa}\right)\right| n \kappa\right\rangle=\left(E_{n^{\prime} \kappa^{\prime}}+E_{n \kappa}\right)\left\langle n^{\prime} \kappa^{\prime}|Q| n \kappa\right\rangle .
\end{aligned}
$$

Substituting $Q=r^{s}, i \sigma_{y} r^{s}$ into equation (14) and $Q=\sigma_{z} r^{s}, \sigma_{x} r^{s}$ into equation (15), and using the commutation properties of the Pauli matrices, we obtain [9]

$$
\begin{gathered}
\left(E_{n^{\prime} \kappa^{\prime}}-E_{n \kappa}\right)\left\langle n^{\prime} \kappa^{\prime}\left|r^{s}\right| n \kappa\right\rangle=-s\left\langle n^{\prime} \kappa^{\prime}\left|i \sigma_{y} r^{s-1}\right| n \kappa\right\rangle \\
+\left(\kappa^{\prime}-\kappa\right)\left\langle n^{\prime} \kappa^{\prime}\left|\sigma_{x} r^{s-1}\right| n \kappa\right\rangle \\
\left(E_{n^{\prime} \kappa^{\prime}}-E_{n \kappa}\right)\left\langle n^{\prime} \kappa^{\prime}\left|i \sigma_{y} r^{s}\right| n \kappa\right\rangle=s\left\langle n^{\prime} \kappa^{\prime}\left|r^{s-1}\right| n \kappa\right\rangle \\
-\left(\kappa^{\prime}+\kappa\right)\left\langle n^{\prime} \kappa^{\prime}\left|\sigma_{z} r^{s-1}\right| n \kappa\right\rangle+2 m\left\langle n^{\prime} \kappa^{\prime}\left|\sigma_{x} r^{s}\right| n \kappa\right\rangle \\
\left(E_{n^{\prime} \kappa^{\prime}}+E_{n \kappa}\right)\left\langle n^{\prime} \kappa^{\prime}\left|\sigma_{z} r^{s}\right| n \kappa\right\rangle=s\left\langle n^{\prime} \kappa^{\prime}\left|\sigma_{x} r^{s-1}\right| n \kappa\right\rangle \\
-\left(\kappa^{\prime}-\kappa\right)\left\langle n^{\prime} \kappa^{\prime}\left|i \sigma_{y} r^{s-1}\right| n \kappa\right\rangle+2 m\left\langle n^{\prime} \kappa^{\prime}\left|r^{s}\right| n \kappa\right\rangle \\
+2\left\langle n^{\prime} \kappa^{\prime}\left|\sigma_{z} V r^{s}\right| n \kappa\right\rangle \\
\left(E_{n^{\prime} \kappa^{\prime}}+E_{n \kappa}\right)\left\langle n^{\prime} \kappa^{\prime}\left|\sigma_{x} r^{s}\right| n \kappa\right\rangle=-s\left\langle n^{\prime} \kappa^{\prime}\left|\sigma_{z} r^{s-1}\right| n \kappa\right\rangle \\
+\left(\kappa^{\prime}+\kappa\right)\left\langle n^{\prime} \kappa^{\prime}\left|r^{s-1}\right| n \kappa\right\rangle+2\left\langle n^{\prime} \kappa^{\prime}\left|\sigma_{x} V r^{s}\right| n \kappa\right\rangle
\end{gathered}
$$

In the next sections, we apply these equations for calculations of the average values of various physical quantities as well as for calculations of various higher-order corrections.

\section{Application of the virial relations for evaluation of the average values}

Let consider equations (16)-(19) for the Coulomb field $(V(r)=-\alpha Z / r)$ and for diagonal matrix elements $\left(n^{\prime} \kappa^{\prime}=n \kappa\right)$. Denoting

$$
A^{s}=\int_{0}^{\infty} d r r^{s}\left(G_{n \kappa}^{2}+F_{n \kappa}^{2}\right),
$$




$$
\begin{aligned}
B^{s} & =\int_{0}^{\infty} d r r^{s}\left(G_{n \kappa}^{2}-F_{n \kappa}^{2}\right), \\
C^{s} & =2 \int_{0}^{\infty} d r r^{s} G_{n \kappa} F_{n \kappa},
\end{aligned}
$$

we obtain $[2,7,9]$

$$
\begin{aligned}
2 m A^{s}-2 E_{n \kappa} B^{s} & =2 \alpha Z B^{s-1}-s C^{s-1}, \\
2 m C^{s} & =-s A^{s-1}+2 \kappa B^{s-1}, \\
2 E_{n \kappa} C^{s} & =2 \kappa A^{s-1}-s B^{s-1}-2 \alpha Z C^{s-1} .
\end{aligned}
$$

From these equations, one easily finds

$$
-\left[(s+1) E_{n \kappa}+2 \kappa m\right] A^{s}+\left[(s+1) m+2 \kappa E_{n \kappa}\right] B^{s}+2 \alpha Z m C^{s}=0
$$

Using equations (23)-(26) for $s=0,1$, we obtain

$$
B^{0}=\frac{E_{n \kappa}}{m}, \quad C^{0}=\frac{\kappa}{\alpha Z} \frac{m^{2}-E_{n \kappa}^{2}}{m^{2}}, \quad B^{-1}=\frac{m}{\alpha Z} \frac{m^{2}-E_{n \kappa}^{2}}{m^{2}} .
$$

In addition, according to the Hellmann-Feynman theorem, we have

$$
\frac{\partial E_{n \kappa}}{\partial \kappa}=\left\langle n \kappa\left|\frac{\partial H_{\kappa}}{\partial \kappa}\right| n \kappa\right\rangle=\left\langle n \kappa\left|\frac{\sigma_{x}}{r}\right| n \kappa\right\rangle .
$$

It yields

$$
C^{-1}=\frac{\partial E_{n \kappa}}{\partial \kappa}=\frac{(\alpha Z)^{2} \kappa m}{N^{3} \gamma},
$$

where $N=\sqrt{\left(\gamma+n_{r}\right)^{2}+(\alpha Z)^{2}}, \gamma=\sqrt{\kappa^{2}-(\alpha Z)^{2}}$, and $n_{r}=n-|\kappa|$. The derivative with respect to $\kappa$ in equation (29) must be taken at a fixed $n_{r}$. Using the formulas for $B^{0}, C^{0}$, $B^{-1}$, and $C^{-1}$ given above and reccurence equations (23)-(26), we can calculate the integrals $A^{s}, B^{s}$, and $C^{s}$ for any integer $s$. Explicit formulas for these calculations were derived in [7]. The formulas expressing the integrals $A^{s+1}, B^{s+1}$, and $C^{s+1}$ in terms of the integrals $A^{s}, B^{s}$, and $C^{s}$ are

$$
\begin{aligned}
A^{s+1}= & \left\{2 \alpha Z E_{n \kappa}(s+1) A^{s}+2 \alpha Z m(s+2) B^{s}\right. \\
& \left.-(s+1)\left[s m+2\left(m+\kappa E_{n \kappa}\right)\right] C^{s}\right\}\left\{2(s+2)\left(m^{2}-E_{n \kappa}^{2}\right)\right\}^{-1}, \\
B^{s+1}= & \left\{2 \alpha Z m(s+1) A^{s}+2 \alpha Z E_{n \kappa}(s+2) B^{s}\right. \\
& \left.-(s+1)\left[2 \kappa m+(s+2) E_{n \kappa}\right] C^{s}\right\}\left\{2(s+2)\left(m^{2}-E_{n \kappa}^{2}\right)\right\}^{-1}, \\
C^{s+1}= & \frac{1}{2 m}\left[2 \kappa B^{s}-(s+1) A^{s}\right] .
\end{aligned}
$$

The reversed formulas are

$$
A^{s}=\frac{4 \alpha Z(s+2)\left(m B^{s+1}-E_{n \kappa} A^{s+1}\right)}{(s+1)\left[(s+1)^{2}-4 \gamma^{2}\right]}+\frac{(s+1) m+2 \kappa E_{n \kappa}}{\alpha Z m\left[(s+1)^{2}-4 \gamma^{2}\right]}
$$




$$
\begin{aligned}
& \times\left\{\left[(s+2) m+2 \kappa E_{n \kappa}\right] B^{s+1}-\left[(s+2) E_{n \kappa}+2 \kappa m\right] A^{s+1}\right\}, \\
B^{s}= & \frac{4 \alpha Z\left(m A^{s+1}-E_{n \kappa} B^{s+1}\right)}{(s+1)^{2}-4 \gamma^{2}}-\frac{(s+1) E_{n \kappa}+2 \kappa m}{\alpha Z m\left[(s+1)^{2}-4 \gamma^{2}\right]} \\
& \times\left\{\left[(s+2) E_{n \kappa}+2 \kappa m\right] A^{s+1}-\left[(s+2) m+2 \kappa E_{n \kappa}\right] B^{s+1}\right\}, \\
C^{s}= & \frac{1}{2 \alpha Z m}\left[(s+1) E_{n \kappa}+2 \kappa m\right] A^{s}-\frac{1}{2 \alpha Z m}\left[(s+1) m+2 \kappa E_{n \kappa}\right] B^{s} .
\end{aligned}
$$

Employing these formulas, one easily finds

$$
\begin{aligned}
C^{1}= & \frac{2 \kappa E_{n \kappa}-m}{2 m^{2}}, \\
A^{-1}= & \frac{\alpha Z m}{\gamma N^{3}}\left(\kappa^{2}+n_{r} \gamma\right) \\
B^{-1}= & \frac{m^{2}-E_{n \kappa}^{2}}{\alpha Z m} \\
A^{-2}= & \frac{2(\alpha Z)^{2} \kappa\left[2 \kappa\left(\gamma+n_{r}\right)-N\right] m^{2}}{N^{4}\left(4 \gamma^{2}-1\right) \gamma} \\
B^{-2}= & \frac{2(\alpha Z)^{2}\left[2 \gamma^{2} N-\kappa\left(\gamma+n_{r}\right)\right] m^{2}}{N^{4}\left(4 \gamma^{2}-1\right) \gamma}, \\
C^{-2}= & \frac{2(\alpha Z)^{3}\left[2 \kappa\left(\gamma+n_{r}\right)-N\right] m^{2}}{N^{4}\left(4 \gamma^{2}-1\right) \gamma}, \\
A^{-3}= & \frac{2(\alpha Z)^{3} m^{3}}{N^{5}\left(4 \gamma^{2}-1\right) \gamma\left(\gamma^{2}-1\right)} \\
& \times\left[N^{2}\left(1+2 \gamma^{2}\right)-3 \kappa N\left(\gamma+n_{r}\right)+3(\alpha Z)^{2}\left(N^{2}-\kappa^{2}\right)\right] .
\end{aligned}
$$

It should be noted here that the integral $A^{-3}$ exists only for $|\kappa| \geq 2$. The integral $C^{1}$ occurs in calculations of the bound-electron $g$ factor. The integrals $C^{-2}$ and $A^{-3}$ occur in calculations of the magnetic dipole and electric quadrupole hyperfine splitting, respectively, (see [11] for details). Formulas (27)-(29) and (36)-(41) were also employed in calculations of the recoil corrections to the atomic energy levels $[12,13]$.

\section{Application of the virial relations for calculations of higher-order corrections}

In calculations of higher-order corrections to various physical quantities one needs to evaluate the sums

$$
\left|i, s, \kappa^{\prime}, n \kappa\right\rangle \equiv \sum_{n^{\prime}}^{\left(E_{n^{\prime} \kappa^{\prime}} \neq E_{n \kappa}\right)} \frac{\left|n^{\prime} \kappa^{\prime}\right\rangle\left\langle n^{\prime} \kappa^{\prime}\left|R_{i}^{s}\right| n \kappa\right\rangle}{E_{n \kappa}-E_{n^{\prime} \kappa^{\prime}}},
$$

where $R_{1}^{s}=r^{s}, R_{2}^{s}=\sigma_{z} r^{s}, R_{3}^{s}=\sigma_{x} r^{s}$, and $R_{4}^{s}=i \sigma_{y} r^{s}$. For instance, to derive the first-order correction to the hydrogenic wave function due to the magnetic dipole hyperfine interaction, 
we need to evaluate the expression (43) for $R_{3}^{-2}$. Let us consider how virial relations (16)(19) can be employed for calculations of these sums in the case of a hydrogenlike atom $(V(r)=-\alpha Z / r)$. For this case, equations (16)-(19) can be rewritten in the following form

$$
\begin{aligned}
\left(E_{n \kappa}-E_{n^{\prime} \kappa^{\prime}}\right) A_{n^{\prime} \kappa^{\prime}, n \kappa}^{s}= & s D_{n^{\prime} \kappa^{\prime}, n \kappa}^{s-1}+\left(\kappa-\kappa^{\prime}\right) C_{n^{\prime} \kappa^{\prime}, n \kappa}^{s-1}, \\
\left(E_{n \kappa}-E_{n^{\prime} \kappa^{\prime}}\right) D_{n^{\prime} \kappa^{\prime}, n \kappa}^{s}= & -2 m C_{n^{\prime} \kappa^{\prime}, n \kappa}^{s}-s A_{n^{\prime} \kappa^{\prime}, n \kappa}^{s}+\left(\kappa^{\prime}+\kappa\right) B_{n^{\prime} \kappa^{\prime}, n \kappa}^{s-1}, \\
\left(E_{n \kappa}-E_{n^{\prime} \kappa^{\prime}}\right) B_{n^{\prime} \kappa^{\prime}, n \kappa}^{s}= & -2 m A_{n^{\prime} \kappa^{\prime}, n \kappa}^{s}+2 E_{n \kappa} B_{n^{\prime} \kappa^{\prime}, n \kappa}^{s}-s C_{n^{\prime} \kappa^{\prime}, n \kappa}^{s-1} \\
& +\left(\kappa^{\prime}-\kappa\right) D_{n^{\prime} \kappa^{\prime}, n \kappa}^{s-1}+2 \alpha Z B_{n^{\prime} \kappa^{\prime}, n \kappa}^{s-1}, \\
\left(E_{n \kappa}-E_{n^{\prime} \kappa^{\prime}}\right) C_{n^{\prime} \kappa^{\prime}, n \kappa}^{s}= & 2 E_{n \kappa} C_{n^{\prime} \kappa^{\prime}, n \kappa}^{s}-\left(\kappa^{\prime}+\kappa\right) A_{n^{\prime} \kappa^{\prime}, n \kappa}^{s}+s B_{n^{\prime} \kappa^{\prime}, n \kappa}^{s-1} \\
& +2 \alpha Z C_{n^{\prime} \kappa^{\prime}, n \kappa}^{s-1},
\end{aligned}
$$

where

$$
\begin{aligned}
& A_{n^{\prime} \kappa^{\prime}, n \kappa}^{s}=\int_{0}^{\infty} d r r^{s}\left(G_{n^{\prime} \kappa^{\prime}} G_{n \kappa}+F_{n^{\prime} \kappa^{\prime}} F_{n \kappa}\right), \\
& B_{n^{\prime} \kappa^{\prime}, n \kappa}^{s}=\int_{0}^{\infty} d r r^{s}\left(G_{n^{\prime} \kappa^{\prime}} G_{n \kappa}-F_{n^{\prime} \kappa^{\prime}} F_{n \kappa}\right), \\
& C_{n^{\prime} \kappa^{\prime}, n \kappa}^{s}=\int_{0}^{\infty} d r r^{s}\left(G_{n^{\prime} \kappa^{\prime}} F_{n \kappa}+F_{n^{\prime} \kappa^{\prime}} G_{n \kappa}\right), \\
& D_{n^{\prime} \kappa^{\prime}, n \kappa}^{s}=\int_{0}^{\infty} d r r^{s}\left(G_{n^{\prime} \kappa^{\prime}} F_{n \kappa}-F_{n^{\prime} \kappa^{\prime}} G_{n \kappa}\right) .
\end{aligned}
$$

From equations (44)-(47), we obtain

$$
\begin{aligned}
\left(E_{n \kappa}-\right. & \left.E_{n^{\prime} \kappa^{\prime}}\right)\left(E_{n \kappa} D_{n^{\prime} \kappa^{\prime}, n \kappa}^{s}+m C_{n^{\prime} \kappa^{\prime}, n \kappa}^{s}\right) \\
= & {\left[-s E_{n \kappa}-m\left(\kappa^{\prime}+\kappa\right)\right] A_{n^{\prime} \kappa^{\prime}, n \kappa}^{s-1}+\left[s m+E_{n \kappa}\left(\kappa^{\prime}+\kappa\right)\right] B_{n^{\prime} \kappa^{\prime}, n \kappa}^{s-1} } \\
& +2 \alpha Z m C_{n^{\prime} \kappa^{\prime}, n \kappa}^{s-1} .
\end{aligned}
$$

For $n \kappa=n^{\prime} \kappa^{\prime}$, this equation turns into equation (26).

Let us consider first the case $\kappa=\kappa^{\prime}$. Taking into account that $A_{n^{\prime} \kappa, n \kappa}^{0}=\delta_{n^{\prime} n}$, we obtain

$$
\begin{aligned}
B_{n^{\prime} \kappa, n \kappa}^{0}= & \frac{1}{m}\left(E_{n \kappa}-E_{n^{\prime} \kappa}\right)\left(E_{n \kappa} D_{n^{\prime} \kappa, n \kappa}^{1}+m C_{n^{\prime} \kappa, n \kappa}^{1}\right. \\
& \left.+\alpha Z D_{n^{\prime} \kappa, n \kappa}^{0}-\kappa B_{n^{\prime} \kappa, n \kappa}^{0}\right)+\frac{E_{n \kappa}}{m} \delta_{n n^{\prime}} .
\end{aligned}
$$

Multiplying this equation with $\left|n^{\prime} \kappa\right\rangle$ and summing over $n^{\prime}$, we derive

$$
\begin{aligned}
|2,0, \kappa, n \kappa\rangle= & \frac{1}{m}(I-|n \kappa\rangle\langle n \kappa|) \\
& \times\left(E_{n \kappa} i \sigma_{y} r+m \sigma_{x} r+\alpha Z i \sigma_{y}-\kappa \sigma_{z}\right)|n \kappa\rangle,
\end{aligned}
$$

where $I$ is the identity operator. From equations (52) and (54), we can derive the sum $|3,0, \kappa, n \kappa\rangle$. Then, using equations (45)-(47) and (52), we can calculate all the sums $|i, s, \kappa, n \kappa\rangle$ 
for $i=1,2,3$ and $s=0,1,2, \ldots$. In particular, for the sum $|3,1, \kappa, n \kappa\rangle$ that occurs in calculations of various corrections to the bound-electron $g$ factor, we find

$$
\begin{aligned}
|3,1, \kappa, n \kappa\rangle= & \frac{\kappa}{m^{2}}(I-|n \kappa\rangle\langle n \kappa|) \\
& \times\left[\left(E_{n \kappa}-\frac{m}{2 \kappa}\right) r i \sigma_{y}+m r \sigma_{x}+\alpha Z i \sigma_{y}-\kappa \sigma_{z}\right]|n \kappa\rangle .
\end{aligned}
$$

The sums $|4, s, \kappa, n \kappa\rangle$ for $s \neq-1$ can be calculated by employing equation (44). The sum $|4,-1, \kappa, n \kappa\rangle$ is easily derived from the relation

$$
D_{n^{\prime} \kappa, n \kappa}^{-1}=\left(E_{n \kappa}-E_{n^{\prime} \kappa}\right)\left\langle n^{\prime} \kappa|\ln r| n \kappa\right\rangle,
$$

which is obtained by differentiation of equation (44) with respect to $s$.

Let us consider now the case $\kappa^{\prime} \neq \kappa$. From equation (44) we find

$$
\left|3,-1, \kappa^{\prime}, n \kappa\right\rangle=\frac{1}{\kappa-\kappa^{\prime}}|n \kappa\rangle .
$$

Then, using equations (44)-(47) and (52), we can calculate all the sums $\left|i, s, \kappa^{\prime}, n \kappa\right\rangle$ for $i=1,2,3,4$ and $s=-2,-3,-4, \ldots$ (if, of course, the corresponding sum exists). For the sum $\left|3,-2, \kappa^{\prime}, n \kappa\right\rangle$ that occurs in calculations of various corrections to the hyperfine splitting, we find

$$
\begin{aligned}
\left|3,-2, \kappa^{\prime}, n \kappa\right\rangle= & \left\{\left[1-\left(\kappa-\kappa^{\prime}\right)^{2}\right]\left[1-\left(\kappa+\kappa^{\prime}\right)^{2}\right]+4(\alpha Z)^{2}\right\}^{-1} \\
& \times\left[[ 1 - ( \kappa + \kappa ^ { \prime } ) ^ { 2 } ] \left(\frac{4 \alpha Z m}{\kappa^{2}-\kappa^{\prime 2}}+\left(\kappa^{\prime}-\kappa\right) r^{-1}+r^{-1} \sigma_{z}\right.\right. \\
& \left.-\frac{2}{\kappa+\kappa^{\prime}}\left(m \sigma_{x}+E_{n \kappa} i \sigma_{y}\right)\right)+\frac{4 \alpha Z\left[\left(\kappa^{\prime}+\kappa\right) m-E_{n \kappa}\right]}{\kappa-\kappa^{\prime}} \\
& \left.+2 \alpha Z\left[\sigma_{x} r^{-1}+\left(\kappa^{\prime}+\kappa\right) r^{-1} i \sigma_{y}\right]\right]|n \kappa\rangle .
\end{aligned}
$$

For $\kappa= \pm \kappa^{\prime}$, the corresponding sums can be calculated by taking the limit $\kappa^{\prime} \rightarrow \pm \kappa$. So, taking the limit $\kappa^{\prime} \rightarrow \kappa$ in equation (44), we obtain

$$
|3,-1, \kappa, n \kappa\rangle=\frac{\partial}{\partial \kappa}|n \kappa\rangle,
$$

where, as in (29), the derivative with respect to $\kappa$ must be taken at a fixed $n_{r}$. Then, the other sums with $\kappa^{\prime}=\kappa$ and $s=-1,-2,-3, \ldots$ can be calculated by using equations (44)-(47) and (52). In particular, we obtain

$$
\begin{aligned}
|3,-2, \kappa, n \kappa\rangle= & \frac{1}{4(\alpha Z)^{2}+\left(1-4 \kappa^{2}\right)}\left[2 \alpha Z \sigma_{x} r^{-1}+4 \alpha Z \kappa i \sigma_{y} r^{-1}\right. \\
& +\left(1-4 \kappa^{2}\right) \sigma_{z} r^{-1}-\frac{\left(1-4 \kappa^{2}\right)}{\kappa}\left(E_{n \kappa} i \sigma_{y}+m \sigma_{x}\right) \\
& \left.-\frac{2(\alpha Z)^{3} \kappa m}{N^{3} \gamma}\right]|n \kappa\rangle-\frac{2 \alpha Z\left(2 E_{n \kappa}-\frac{m}{\kappa}\right)}{4(\alpha Z)^{2}+\left(1-4 \kappa^{2}\right)} \frac{\partial}{\partial \kappa}|n \kappa\rangle .
\end{aligned}
$$


The case $\kappa^{\prime}=-\kappa$ can be considered in the same way.

Concluding this section, we give the explicit formulas for $\frac{\partial}{\partial \kappa}|n \kappa\rangle$ for the $1 s$ and $2 s$ states [14]. For the $1 s$ state:

$$
\frac{\partial}{\partial \kappa}|n \kappa\rangle=\left(\begin{array}{c}
\tilde{G}_{1 s}(r) \\
\tilde{F}_{1 s}(r)
\end{array}\right)
$$

where

$$
\begin{aligned}
\tilde{G}_{1 s}(r)= & \frac{k}{\sqrt{1-\gamma}} \exp (-t / 2) t^{\gamma}\left(\frac{\psi(2 \gamma+1)}{\gamma}+(\gamma+1)\right. \\
& \left.-\frac{1}{2 \gamma}-\frac{t}{2}-\frac{1}{\gamma} \ln t\right) \\
\tilde{F}_{1 s}(r)= & -\frac{k}{\sqrt{1+\gamma}} \exp (-t / 2) t^{\gamma}\left(\frac{\psi(2 \gamma+1)}{\gamma}+(\gamma+1)\right. \\
& \left.+\frac{1}{2 \gamma}-\frac{t}{2}-\frac{1}{\gamma} \ln t\right), \\
t= & \frac{2 \alpha Z m r}{N}, \quad k=\frac{(2 \alpha Z)^{\frac{3}{2}} m^{\frac{1}{2}}}{2 \sqrt{2 \Gamma(2 \gamma+1)}},
\end{aligned}
$$

$\Gamma(x)$ is the gamma-function, and $\psi(x)=\frac{d}{d x} \ln \Gamma(x)$. For the $2 s$ state:

$$
\frac{\partial}{\partial \kappa}|n \kappa\rangle=\left(\begin{array}{c}
\tilde{G}_{2 s}(r) \\
\tilde{F}_{2 s}(r)
\end{array}\right)
$$

where

$$
\begin{aligned}
\tilde{G}_{2 s}(r)= & k^{\prime} \exp \left(-\frac{t}{2}\right) t^{\gamma} \frac{\sqrt{2+N}}{N^{2}-2}\left\{\frac{t^{2}}{2(N-1)}\right. \\
& -\left(\frac{2 N^{4}-4 N^{3}+5 N^{2}-3 N+2}{2 N(N-1)^{2}}+\frac{2 \psi(2 \gamma+1)}{N-1}\right) t \\
& +\frac{N^{4}-2 N^{3}+N-2}{2(N-1)}+2 N \psi(2 \gamma+1) \\
& \left.+\frac{2}{N-1} t \ln t-2 N \ln t\right\} \\
\tilde{F}_{2 s}(r)= & -k^{\prime} \exp \left(-\frac{t}{2}\right) t^{\gamma} \frac{\sqrt{2-N}}{N^{2}-2}\left\{\frac{t^{2}}{2(N-1)}\right. \\
& -\left(\frac{2 N^{4}-2 N^{3}+N^{2}+3 N-2}{2 N(N-1)^{2}}+\frac{2 \psi(2 \gamma+1)}{N-1}\right) t \\
& +\frac{N^{5}+5 N^{2}-8 N-4}{2 N(N-1)}+2(N+2) \psi(2 \gamma+1) \\
& \left.+\frac{2}{N-1} t \ln t-2(N+2) \ln t\right\},
\end{aligned}
$$




$$
t=\frac{2 \alpha Z m r}{N}, \quad k^{\prime}=\frac{\sqrt{\Gamma(2 \gamma+2)}}{\Gamma(2 \gamma+1)} \frac{1}{\sqrt{8 N(N+1)}}\left(\frac{2 \alpha Z m}{N}\right)^{\frac{1}{2}}
$$

\section{Calculations of the bound-electron $g$ factor and the hyperfine splitting in $\mathrm{H}$-like atoms}

For the last few years a significant progress was achieved in calculations of the bound-electron $g$ factor and the hyperfine splitting in H-like atoms. Formulas (55) and (60) were extensively employed in these calculations.

In [15], a complete $\alpha Z$-dependence formula for the recoil correction of order $m / M$ to the $g$ factor of an H-like atom was derived. According to this formula, which was confirmed by an independent derivation in [16], the recoil correction is given by $(e<0)$

$$
\begin{aligned}
\Delta g= & \frac{1}{\mu_{0} m_{a}} \frac{i}{2 \pi M} \int_{-\infty}^{\infty} d \omega\left[\frac{\partial}{\partial \mathcal{H}}\langle\tilde{a}|\left[p^{k}-D^{k}(\omega)+e A_{\mathrm{cl}}^{k}\right]\right. \\
& \left.\times \tilde{G}\left(\omega+\tilde{E}_{a}\right)\left[p^{k}-D^{k}(\omega)+e A_{\mathrm{cl}}^{k}\right]|\tilde{a}\rangle\right]_{\mathcal{H}=0} .
\end{aligned}
$$

Here $\mu_{0}$ is the Bohr magneton, $m_{a}$ is the angular momentum projection of the state $a$, $p^{k}=-i \nabla^{k}$ is the momentum operator, $\mathbf{A}_{\mathrm{cl}}=[\mathcal{H} \times \mathbf{r}] / 2$ is the vector potential of the homogeneous magnetic field $\mathcal{H}$ directed along the $z$ axis, $D^{k}(\omega)=-4 \pi \alpha Z \alpha^{l} D^{l k}(\omega)$,

$$
D^{i l}(\omega, \mathbf{r})=-\frac{1}{4 \pi}\left\{\frac{\exp (i|\omega| r)}{r} \delta_{i l}+\nabla^{i} \nabla^{l} \frac{(\exp (i|\omega| r)-1)}{\omega^{2} r}\right\}
$$

is the transverse part of the photon propagator in the Coulomb gauge. The tilde sign indicates that the related quantity (the wave function, the energy, and the Coulomb-Green function $\tilde{G}(\omega)=\sum_{\tilde{n}}|\tilde{n}\rangle\langle\tilde{n}|\left[\omega-\tilde{E}_{n}(1-i 0)\right]^{-1}$ ) must be calculated at the presence of the homogeneous magnetic field $\mathcal{H}$ directed along the $z$ axis. In equation (67) and below, the summation over the repeated indices $(k=1,2,3)$, which enumerate components of the threedimensional vectors, is implicit. For the practical calculations, this expression is conveniently represented by the sum of the lower-order term and the higher-order term, $\Delta g=\Delta g_{\mathrm{L}}+\Delta g_{\mathrm{H}}$, where

$$
\begin{aligned}
\Delta g_{\mathrm{L}}= & \frac{1}{\mu_{0} m_{a}} \frac{1}{2 M}\left[\frac{\partial}{\partial \mathcal{H}}\left\langle\tilde{a}\left|\left\{\mathbf{p}^{2}-\frac{\alpha Z}{r}[(\boldsymbol{\alpha} \cdot \mathbf{p})+(\boldsymbol{\alpha} \cdot \mathbf{n})(\mathbf{n} \cdot \mathbf{p})]\right\}\right| \tilde{a}\right\rangle\right]_{\mathcal{H}=0} \\
& -\frac{1}{m_{a}} \frac{m}{M}\left\langle a\left|\left([\mathbf{r} \times \mathbf{p}]_{z}-\frac{\alpha Z}{2 r}[\mathbf{r} \times \boldsymbol{\alpha}]_{z}\right)\right| a\right\rangle, \\
\Delta g_{\mathrm{H}}= & \frac{1}{\mu_{0} m_{a}} \frac{i}{2 \pi M} \int_{-\infty}^{\infty} d \omega\left[\frac{\partial}{\partial \mathcal{H}}\langle\tilde{a}|\left(D^{k}(\omega)-\frac{\left[p^{k}, V\right]}{\omega+i 0}\right)\right. \\
& \left.\times \tilde{G}\left(\omega+\tilde{E}_{a}\right)\left(D^{k}(\omega)+\frac{\left[p^{k}, V\right]}{\omega+i 0}\right)|\tilde{a}\rangle\right]_{\mathcal{H}=0},
\end{aligned}
$$


where $V(r)=-\alpha Z / r$ is the Coulomb potential induced by the nucleus and $\mathbf{n}=\mathbf{r} / r$. The lower-order term can be calculated analytically by employing formula (55) and the formulas for the average values presented above. Let us consider this calculation in details [15]. According to equation (69), the lower-order term is the sum of two contributions, $\Delta g_{\mathrm{L}}=\Delta g_{\mathrm{L}}^{(1)}+\Delta g_{\mathrm{L}}^{(2)}$. The first contribution is

$$
\Delta g_{\mathrm{L}}^{(1)}=\frac{1}{\mu_{0} m_{a} \mathcal{H}} \frac{1}{M}\langle\delta a|\left[\mathbf{p}^{2}-\frac{\alpha Z}{r}(\boldsymbol{\alpha} \cdot \mathbf{p}+(\boldsymbol{\alpha} \cdot \mathbf{n})(\mathbf{n} \cdot \mathbf{p})]|a\rangle,\right.
$$

where $\delta a$ is the first-order correction to the electron wave function due to interaction with the homogeneous magnetic field. Taking into account that $\mathbf{p}^{2}=(\boldsymbol{\alpha} \cdot \mathbf{p})^{2}$ and $(\boldsymbol{\alpha} \cdot \mathbf{p})=H-\beta m-V$, one easily obtains

$$
\begin{aligned}
\left\langle\delta a\left|\mathbf{p}^{2}\right| a\right\rangle= & \left\langle\delta a\left|\left(E_{a}+\beta m-V\right)\left(E_{a}-\beta m-V\right)\right| a\right\rangle \\
& +i\langle\delta a|(\boldsymbol{\alpha} \cdot \boldsymbol{\nabla} V)| a\rangle .
\end{aligned}
$$

The second term in equation (71) can be transformed as (see, e.g., [13])

$$
\begin{aligned}
-\left\langle\delta a\left|\frac{\alpha Z}{r}[\boldsymbol{\alpha} \cdot \mathbf{p}+(\boldsymbol{\alpha} \cdot \mathbf{n})(\mathbf{n} \cdot \mathbf{p})]\right| a\right\rangle= & -\langle\delta a| \frac{\alpha Z}{r}\left[2 E_{a}-2 \beta m-2 V\right. \\
& \left.+\frac{i}{r}(\boldsymbol{\alpha} \cdot \mathbf{n})(\beta \kappa+1)\right]|a\rangle .
\end{aligned}
$$

The wave function correction $|\delta a\rangle$ is defined by

$$
|\delta a\rangle=\sum_{n}^{E_{n} \neq E_{a}} \frac{|n\rangle\left\langle n|| e\left|\boldsymbol{\alpha} \cdot \mathbf{A}_{\mathrm{cl}}\right| a\right\rangle}{E_{a}-E_{n}}
$$

Since the operator sandwiched between $|a\rangle$ and $|\delta a\rangle$ in equation for $\Delta g_{\mathrm{L}}^{(1)}$ conserves the angular quantum numbers, we need only that component of $|\delta a\rangle$ which has the same angular quantum numbers as the unperturbed state $|a\rangle$. Using formula (55), we easily find

$$
|\delta a\rangle_{\kappa m_{a}}=\left(\begin{array}{c}
X(r) \Omega_{\kappa m_{a}}(\mathbf{n}) \\
i Y(r) \Omega_{-\kappa m_{a}}(\mathbf{n})
\end{array}\right)
$$

where

$$
\begin{aligned}
X(r) & =b_{0}\left\{\left[\frac{2 m \kappa-m+2 \kappa E_{a}}{2 m^{2}} r+\frac{\alpha Z}{m^{2}} \kappa\right] f(r)+\frac{\kappa-2 \kappa^{2}}{2 m^{2}} g(r)\right\}, \\
Y(r) & =b_{0}\left\{\left[\frac{2 m \kappa+m-2 \kappa E_{a}}{2 m^{2}} r-\frac{\alpha Z}{m^{2}} \kappa\right] g(r)+\frac{\kappa+2 \kappa^{2}}{2 m^{2}} f(r)\right\}, \\
b_{0} & =-\frac{e}{2} \mathcal{H} \frac{\kappa}{j(j+1)} m_{a},
\end{aligned}
$$

$g(r)$ and $f(r)$ are the radial parts of the unperturbed wave function defined above. Integrating over the angular variables in equations (72) and (73), we find

$$
\Delta g_{\mathrm{L}}^{(1)}=\frac{\kappa}{j(j+1)} \frac{m}{M} \int_{0}^{\infty} d r r^{2}\left\{X(r) g(r)\left[-2 V m-V^{2}+E_{a}^{2}-m^{2}\right]\right.
$$




$$
\begin{aligned}
& +Y(r) f(r)\left[2 V m-V^{2}+E_{a}^{2}-m^{2}\right] \\
& \left.+[X(r) f(r)+Y(r) g(r)] \frac{\alpha Z}{r^{2}} \kappa\right\} .
\end{aligned}
$$

Substituting expressions (76) and (77) into equation (79), we obtain

$$
\begin{aligned}
\Delta g_{\mathrm{L}}^{(1)}= & \frac{\kappa}{j(j+1)} \frac{m}{M}\left\{\alpha Z \frac{2 \kappa E_{a}-m}{m} C^{0}+(\alpha Z)^{2} \frac{\kappa}{m} C^{-1}\right. \\
& +\left(E_{a}^{2}-m^{2}\right) \frac{\kappa}{m} C^{1}+\alpha Z \frac{\kappa^{2}}{2 m^{2}} C^{-2}+\left(E_{a}^{2}-m^{2}\right) \frac{\kappa}{2 m^{2}} A^{0} \\
& -\alpha Z \frac{\kappa^{2}}{m} A^{-1}-(\alpha Z)^{2} \frac{\kappa}{2 m^{2}} A^{-2}-\left(E_{a}^{2}-m^{2}\right) \frac{\kappa^{2}}{m^{2}} B^{0} \\
& \left.+\alpha Z \frac{3 m \kappa-2 \kappa^{2} E_{a}}{2 m^{2}} B^{-1}\right\} .
\end{aligned}
$$

Using the explicit expressions for the integrals $A^{s}, B^{s}$, and $C^{s}$ given above, we obtain

$$
\Delta g_{\mathrm{L}}^{(1)}=\frac{m}{M} \frac{\kappa^{2}}{2 j(j+1)} \frac{m^{2}-E_{a}^{2}}{m^{2}} .
$$

Consider now the contribution $\Delta g_{\mathrm{L}}^{(2)}$ :

$$
\Delta g_{\mathrm{L}}^{(2)}=-\frac{1}{m_{a}} \frac{m}{M}\left\langle a\left|\left(l_{z}-\frac{\alpha Z}{2 r}[\mathbf{r} \times \boldsymbol{\alpha}]_{z}\right)\right| a\right\rangle .
$$

Integrating over the angular variables and employing the explicit expressions for the radial integrals derived above, we obtain

$$
\begin{aligned}
\Delta g_{\mathrm{L}}^{(2)}= & -\frac{m}{M} \frac{1}{2 j(j+1)}\left\{j(j+1)-\frac{3}{4}+l(l+1) \frac{m+E_{a}}{2 m}\right. \\
& \left.+(2 j-l)(2 j-l+1) \frac{m-E_{a}}{2 m}-\kappa^{2} \frac{m^{2}-E_{a}^{2}}{m^{2}}\right\} .
\end{aligned}
$$

For the sum $\Delta g_{\mathrm{L}}=\Delta g_{\mathrm{L}}^{(1)}+\Delta g_{\mathrm{L}}^{(2)}$, we find

$$
\Delta g_{\mathrm{L}}=-\frac{m}{M} \frac{2 \kappa^{2} E_{a}^{2}+\kappa m E_{a}-m^{2}}{2 m^{2} j(j+1)} .
$$

To the two lowest orders in $\alpha Z$, we have

$$
\Delta g_{\mathrm{L}}=-\frac{m}{M} \frac{1}{j(j+1)}\left[\kappa^{2}+\frac{\kappa}{2}-\frac{1}{2}-\left(\kappa^{2}+\frac{\kappa}{4}\right) \frac{(\alpha Z)^{2}}{n^{2}}\right] .
$$

For the $1 s$ state, formula (84) yields

$$
\Delta g_{\mathrm{L}}=\frac{m}{M}(\alpha Z)^{2}-\frac{m}{M} \frac{(\alpha Z)^{4}}{3\left[1+\sqrt{1-(\alpha Z)^{2}}\right]^{2}} .
$$


The first term in the right-hand side of this equation reproduces the result of $[17,18]$. The higher-order term $\Delta g_{\mathrm{H}}$ was evaluated numerically for the $1 s$ state in [19]. Formula (55) was also extensively used in that evaluation.

Let us consider now the derivation of the nuclear-size correction to the $g$ factor of a low- $Z$ H-like atom [20]. To find this correction, we have to evaluate the expression

$$
\Delta g=\frac{2}{\mu_{0} m_{a} \mathcal{H}} \sum_{n}^{n \neq a} \frac{\langle a|\delta V| n\rangle\left\langle n|| e\left|\boldsymbol{\alpha} \cdot \mathbf{A}_{\mathrm{cl}}\right| a\right\rangle}{E_{a}-E_{n}},
$$

where $\delta V$ determines the deviation of the potential from the pure Coulomb one. Integrating over the angular variables, we obtain

$$
\Delta g=\frac{2 \kappa m}{j(j+1)} \sum_{n^{\prime}}^{n^{\prime} \neq n} \frac{\left\langle n \kappa|\delta V| n^{\prime} \kappa\right\rangle\left\langle n^{\prime} \kappa\left|r \sigma_{x}\right| n \kappa\right\rangle}{E_{n \kappa}-E_{n^{\prime} \kappa}},
$$

where $|n \kappa\rangle$ are the two-component radial wave functions defined above. Substituting expression (55) into this equation, we get

$$
\begin{aligned}
\Delta g= & \frac{2 \kappa^{2}}{j(j+1) m}\left\{\left\langle n \kappa\left|\delta V\left[\left(E_{n \kappa}-\frac{m}{2 \kappa}\right) r i \sigma_{y}+m r \sigma_{x}+\alpha Z i \sigma_{y}-\kappa \sigma_{z}\right]\right| n \kappa\right\rangle\right. \\
& -\left\langle n \kappa\left|\left[\left(E_{n \kappa}-\frac{m}{2 \kappa}\right) r i \sigma_{y}+m r \sigma_{x}+\alpha Z i \sigma_{y}-\kappa \sigma_{z}\right]\right| n \kappa\right\rangle \\
& \times\langle n \kappa|\delta V| n \kappa\rangle\} .
\end{aligned}
$$

We assume that the nuclear charge distribution is described by a spherically symmetric density $\rho(\mathbf{r})=\rho(r)$, which is normalized by the equation

$$
\int d \mathbf{r} \rho(\mathbf{r})=1
$$

The Poisson equation gives

$$
\Delta(\delta V)(\mathbf{r})=4 \pi \alpha Z[\rho(\mathbf{r})-\delta(\mathbf{r})],
$$

where $\Delta$ is the Laplacian. When integrated with $\delta V$, the radial functions $g(r)$ and $f(r)$ can be approximated by the lowest order term of the expansion in powers of $r$. It follows that we have to evaluate the integral

$$
I=\int_{0}^{\infty} d r r^{2} r^{2 \gamma-2} \delta V
$$

Using the identity

$$
r^{\beta}=\frac{1}{(\beta+2)(\beta+3)} \Delta r^{\beta+2}
$$


and integrating by parts, we find

$$
\begin{array}{r}
I=\int_{0}^{\infty} d r r^{2} \frac{1}{2 \gamma(2 \gamma+1)} \Delta r^{2 \gamma} \delta V=\int_{0}^{\infty} d r r^{2} \frac{1}{2 \gamma(2 \gamma+1)} r^{2 \gamma} \Delta(\delta V) \\
=\frac{4 \pi \alpha Z}{2 \gamma(2 \gamma+1)} \int_{0}^{\infty} d r r^{2} r^{2 \gamma} \rho(r)=\frac{\alpha Z}{2 \gamma(2 \gamma+1)}\left\langle r^{2 \gamma}\right\rangle_{\mathrm{nuc}}
\end{array}
$$

where

$$
\left\langle r^{2 \gamma}\right\rangle_{\mathrm{nuc}}=\int d \mathbf{r} r^{2 \gamma} \rho(r) .
$$

For the correction to the $g$ factor we obtain

$$
\begin{aligned}
\Delta g= & \frac{\kappa^{2}}{j(j+1)} \frac{\Gamma\left(2 \gamma+1+n_{r}\right) 2^{2 \gamma-1}}{\gamma(2 \gamma+1) \Gamma^{2}(2 \gamma+1) n_{r} !(N-\kappa) N^{2 \gamma+2}} \\
& \times\left[\left[n_{r}^{2}+(N-\kappa)^{2}\right]\left(1-2 \kappa \frac{E_{n \kappa}}{m}\right)-2 n_{r}(N-\kappa)\left(\frac{E_{n \kappa}}{m}-2 \kappa\right)\right] \\
& \times(\alpha Z)^{2 \gamma+2} m^{2 \gamma}\left\langle r^{2 \gamma}\right\rangle_{\text {nuc }} .
\end{aligned}
$$

For $n s$-states, which are of particular interest, the expansion of this expression to two lowest orders in $\alpha Z$ yields

$$
\begin{aligned}
\Delta g= & \frac{8}{3 n^{3}}(\alpha Z)^{4} m^{2}\left\langle r^{2}\right\rangle_{\text {nuc }}\left[1+(\alpha Z)^{2}\left(\frac{1}{4}+\frac{12 n^{2}-n-9}{4 n^{2}(n+1)}\right.\right. \\
& \left.\left.+2 \psi(3)-\psi(2+n)-\frac{\left\langle r^{2} \ln (2 \alpha Z m r / n)\right\rangle_{\text {nuc }}}{\left\langle r^{2}\right\rangle_{\text {nuc }}}\right)\right]
\end{aligned}
$$

where $\psi(x)=\frac{d}{d x} \ln \Gamma(x)$. For the $1 s$ state, we have

$$
\Delta g=\frac{8}{3}(\alpha Z)^{4} m^{2}\left\langle r^{2}\right\rangle_{\mathrm{nuc}}\left[1+(\alpha Z)^{2}\left(2-C-\frac{\left\langle r^{2} \ln (2 \alpha Z m r)\right\rangle_{\mathrm{nuc}}}{\left\langle r^{2}\right\rangle_{\mathrm{nuc}}}\right)\right],
$$

where $C=0.57721566490 \ldots$ is the Euler constant. In the non-relativistic limit, we find

$$
\Delta g=\frac{8}{3 n^{3}}(\alpha Z)^{4} m^{2}\left\langle r^{2}\right\rangle_{\mathrm{nuc}}
$$

for $n s$ states and

$$
\Delta g=\frac{2\left(n^{2}-1\right)}{3 n^{5}}(\alpha Z)^{6} m^{2}\left\langle r^{2}\right\rangle_{\text {nuc }}
$$




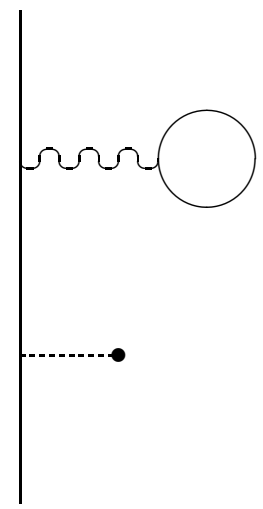

a

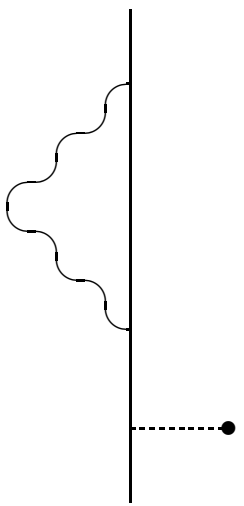

d

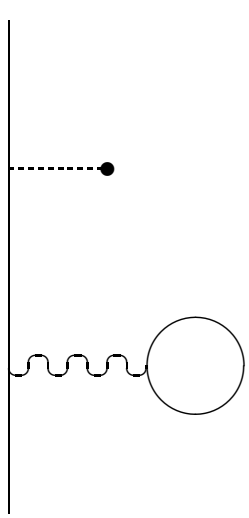

$\mathrm{b}$

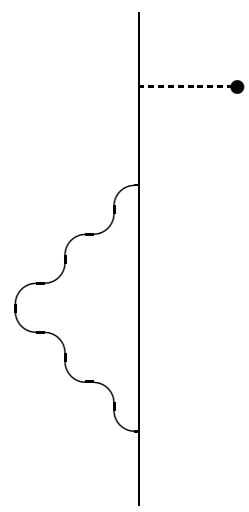

$\mathrm{e}$

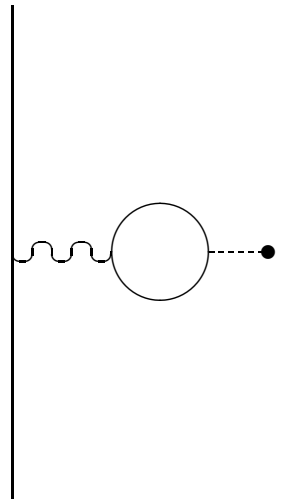

$\mathrm{c}$

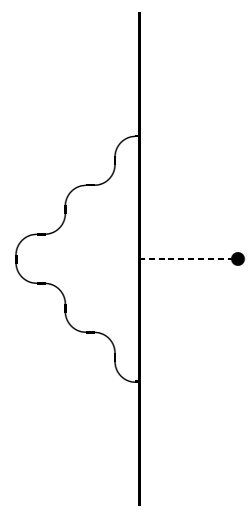

f

Figure 1: The first-order QED corrections to the interaction of the electron with a magnetic field. 
for $n p_{\frac{1}{2}}$ states. In the case of the $1 s$ state, the expression (99) coincides with the related formula in [21]. A similar derivation for the nuclear-size correction to the hyperfne spliting was performed in [22].

In $[23,24]$, formulas $(55)$ and $(60)$ were employed to evaluate analytically the one-loop vacuum-polarization (VP) corrections to the bound-electron $g$ factor and to the hyperfine splitting in low- $Z$ H-like atoms. These corrections are described by diagrams presented in Fig. 1 (a-c), where the dashed line ended by a circle indicates the interaction with a magnetic field. According to [23], for the $1 s$ state, the VP correction to the $g$ factor calculated in the Uehling approximation is given by

$$
\begin{aligned}
\Delta g= & \frac{\alpha}{\pi}\left[-\frac{16}{15}(\alpha Z)^{4}+\frac{5 \pi}{9}(\alpha Z)^{5}\right. \\
& +\left(\frac{16}{15} \ln (2 \alpha Z)-\frac{2012}{525}\right)(\alpha Z)^{6} \\
& \left.+\left(-\frac{5 \pi}{9} \ln (\alpha Z / 2)-\frac{125 \pi}{216}\right)(\alpha Z)^{7}+\cdots\right] .
\end{aligned}
$$

The Uehling correction to the $1 s$ hyperfine splitting is [24]

$$
\begin{aligned}
\Delta E_{\mathrm{hfs}}= & \frac{\alpha}{\pi} E_{\mathrm{F}}\left[\frac{3 \pi}{4}(\alpha Z)+\left(\frac{34}{225}-\frac{8}{15} \ln (2 \alpha Z)\right)(\alpha Z)^{2}\right. \\
& \left.+\left(\frac{539 \pi}{288}-\frac{13 \pi}{24} \ln (\alpha Z / 2)\right)(\alpha Z)^{3}+\cdots\right]
\end{aligned}
$$

where $E_{\mathrm{F}}$ is the Fermi energy. In [25, 26], the one-loop self-energy corrections to the hyperfine splitting and to the bound-electron $g$ factor (Fig. 1 (d-f)) were calculated numerically to a high accuracy. Formulas (55) and (60) were also employed in these calculations. In [27], formula (55) was used to evaluate the nuclear-polarization effect on the bound-electron $g$ factor.

\section{Other applications of the virial relations}

Applications of the virial relations are not restricted only by $\mathrm{H}$-like atoms and by the pure Coulomb field. In [14], formulas (58) and (60) were employed to calculate the interelectronicinteraction corrections to the hyperfine splitting in Li-like ions. In [29], virial relations (16)-(19) with $V(r) \neq-\alpha Z / r$ were used to evaluate the recoil correction to the Lamb shift for an extended nucleus. As an example, let us demonstrate how the virial relations with $V(r) \neq-\alpha Z / r$ can be employed to evaluate the nuclear-size correction of the lowest order in $m R$ ( $R$ is the nuclear charge radius) to the integral

$$
C^{-2}=2 \int_{0}^{\infty} d r r^{-2} G F
$$

for an $n s$ state, which we denote by $|n s\rangle$. This integral occurs in calculations of the hyperfine splitting. We consider virial relations (16)-(19) for the case $n=n^{\prime}, \kappa=\kappa^{\prime}$ and for the 
potential

$$
V(r)=-\frac{\alpha Z}{r}+\delta V(r),
$$

where $\delta V$ determines a deviation of the potential from the pure Coulomb one due to the finite-nuclear-size effect. Using notations (20), (21), and (22), where $G$ and $F$ are calculated for potential (104), from equations (16)-(19) we derive

$$
\begin{gathered}
A^{-2}-2 B^{-2}=\text { "regular terms", } \\
C^{-2}-2\left\langle n s\left|\sigma_{z} r^{-1} V\right| n s\right\rangle=\text { "regular terms", } \\
2 A^{-2}-B^{-2}-2\left\langle n s\left|\sigma_{x} r^{-1} V\right| n s\right\rangle=\text { "regular terms", }
\end{gathered}
$$

where by "regular terms" we denote terms which have the nuclear-size corrections of order $(m R)^{2}$ and higher (it means that their integrands have more regular behaviour at $r \rightarrow 0$ than the integrand of $C^{-2}$ has). From these equations, we easily obtain

$$
\begin{aligned}
C^{-2}= & \frac{2}{3-4(\alpha Z)^{2}}\left\langle n s\left|\left(3 \sigma_{z} r^{-1}-2 \alpha Z \sigma_{x} r^{-1}\right) \delta V\right| n s\right\rangle \\
& + \text { "regular terms". }
\end{aligned}
$$

To the lowest order in $m R$, it follows

$$
\delta C^{-2}=\frac{2}{3-4(\alpha Z)^{2}}\left\langle n s\left|\left(3 \sigma_{z} r^{-1}-2 \alpha Z \sigma_{x} r^{-1}\right) \delta V\right| n s\right\rangle .
$$

An analytical evaluation of this expression to the two lowest orders in $\alpha Z$ yields [28]

$$
\begin{aligned}
\delta C^{-2}= & \frac{4}{n^{3}}(\alpha Z)^{4} m^{3}\langle r\rangle_{\mathrm{nuc}}\left\{1+(\alpha Z)^{2}[2 \psi(3)-\psi(n+1)\right. \\
& \left.\left.-\frac{\langle r \ln (2 \alpha Z m r / n)\rangle_{\mathrm{nuc}}}{\langle r\rangle_{\mathrm{nuc}}}+\frac{8 n-9}{4 n^{2}}+\frac{11}{4}\right]\right\} .
\end{aligned}
$$

The non-relativistic limit is given by

$$
\delta C^{-2} / C^{-2}=-2 \alpha Z m\langle r\rangle_{\text {nuc }} .
$$

Formula (111) coincides with the related expression derived in [11] for the sphere model for the nuclear charge distribution, while the relativistic $n$-independent term in formula (110) differs from the corresponding term that can be derived from the formulas presented in [11]. Since, for the sphere model, the approach developed in [11] provides a more accurate evaluation of the nuclear size correction than the perturbation theory considered here, formula (110) can be improved by replacing the relativistic $n$-independent term with the 
corresponding term derived from [11]. As a result, we obtain [22]

$$
\begin{aligned}
\delta C^{-2}= & \frac{4}{n^{3}}(\alpha Z)^{4} m^{3}\langle r\rangle_{\mathrm{nuc}}\left\{1+(\alpha Z)^{2}[2 \psi(3)-\psi(n+1)\right. \\
& \left.\left.-\frac{\langle r \ln (2 \alpha Z m r / n)\rangle_{\mathrm{nuc}}}{\langle r\rangle_{\mathrm{nuc}}}+\frac{8 n-9}{4 n^{2}}+\frac{839}{750}\right]\right\} .
\end{aligned}
$$

Formulas (110) and (112) differ only by the last constant term.

The virial relations are also helpful for calculations employing finite basis set methods or analytical expressions for the Coulomb-Green function. In particular, they were employed in $[30,31]$ to calculate the nuclear recoil corrections by using the B-spline method for the Dirac equation [32]. In that paper, using the virial relations, the original formulas for the recoil corrections, which contain some integrands with a singular behaviour at $r \rightarrow 0$, were expressed in terms of less singular integrals. As a result, the convergence of the numerical procedure for small $r$ was significantly improved. In [8], the virial relations for diagonal matrix elements were used to construct Rayleigh-Schrödinger expansions for eigenvalues of perturbed radial Dirac equations to arbitrary order.

\section{Conclusion}

In this paper we have considered the derivation of the virial relations for the Dirac equation and their applications for calculations of various physical quantities. It has been demonstrated that the virial relations are a very effective tool for analytical and high-precision numerical calculations of the hyperfine splitting and the bound-electron $g$ factor in H-like

ions. They are also useful for calculations employing finite basis set methods and analytical expressions for the Coulomb-Green function.

\section{Acknowledgments}

Valuable conversations with D.A. Glazov, V.G. Ivanov, U. Jentschura, S.G. Karshenboim, A.V. Nefiodov, A.V. Volotka, and V.A. Yerokhin are gratefully acknowledged. This work was supported in part by RFBR (Grant No. 01-02-17248), by the program "Russian Universities" (Grant No. UR.01.01.072), and by GSI.

\section{References}

[1] J. Hirschfelder: J. Chem. Phys. 33, 1762 (1960)

[2] J.H. Epstein, S.T. Epstein: Am. J. Phys. 30, 266 (1962)

[3] S.T. Epstein, J.H. Epstein, B. Kennedy: J. Math. Phys. 8, 1747 (1967) 
[4] P. Blanchard: J. Phys. B 7, 993 (1974)

[5] V. Fock: Z. Physik 63, 855 (1930)

[6] S.P. Goldman, G.W. Drake: Phys. Rev. A 25, 2877 (1982)

[7] V.M. Shabaev: Vestn. Leningr. Univ. 4, 15 (1984)

[8] E.R. Vrscay, H. Hamidian: Phys. Lett. A 130, 141 (1988)

[9] V.M. Shabaev: J. Phys. B 24, 4479 (1991)

[10] G.W. Drake, S.P. Goldman: Phys. Rev. A23, 2093 (1981)

[11] V.M. Shabaev: J. Phys. B 27, 5825 (1994)

[12] V.M. Shabaev: Theor. Math. Phys. 63, 588 (1985)

[13] V.M. Shabaev, A.N. Artemyev: J. Phys. B 27, 1307 (1994)

[14] M.B. Shabaeva, V.M. Shabaev: Phys. Rev. A 52, 2811 (1995)

[15] V.M. Shabaev: Phys. Rev. A 64, 052104 (2001)

[16] A. Yelkhovsky: E-print, hep-ph/0108091 (2001)

[17] R.N. Faustov: Phys. Lett. B 33, 422 (1970); Nuovo Cimento A 69, 37 (1970)

[18] H. Grotch: Phys. Rev. A 2, 1605 (1970)

[19] V.M. Shabaev, V.A. Yerokhin: Phys. Rev. Lett. 88, 091801 (2002)

[20] D.A. Glazov, V.M. Shabaev: Phys. Lett. A 297, 408 (2002)

[21] S.G. Karshenboim: Phys. Lett. A 266, 380 (2000)

[22] A.V. Volotka, V.M. Shabaev, G. Plunien, G. Soff: to be published

[23] S.G. Karshenboim, V.G. Ivanov, V.M. Shabaev: JETP 93, 477 (2001); Can. J. Phys. 79, 81 (2001)

[24] S.G. Karshenboim, V.G. Ivanov, V.M. Shabaev: JETP 90, 59 (2000); Can. J. Phys. 76, 503 (1998)

[25] V.A. Yerokhin, V.M. Shabaev: Phys. Rev. A 64, 012506 (2001)

[26] V.A. Yerokhin, P. Indelicato, V.M. Shabaev: Phys. Rev. Lett. 89, 143001 (2002)

[27] A.V. Nefiodov, G. Plunien, G. Soff: Phys. Rev. Lett. 89, 081802 (2002).

[28] A.V. Volotka, private communication 
[29] V.M. Shabaev, A.N. Artemyev, T. Beier, G. Plunien, V.A. Yerokhin, G. Soff: Phys. Rev. A 57, 4235 (1998); Phys. Scr. T 80, 493 (1999)

[30] A.N. Artemyev, V.M. Shabaev, V.A. Yerokhin: Phys. Rev. A 52, 1884 (1995); J. Phys. B 28, $5201(1995)$

[31] V.M. Shabaev, A.N. Artemyev, T. Beier, G. Soff: J. Phys. B 31, L337 (1998)

[32] W.R. Johnson, S.A. Blundell, J. Sapirstein: Phys. Rev. A 37, 307 (1988) 